\title{
Exotic Solids Made by Elastic Metamaterials
}

\author{
Ying $\mathrm{Wu}$, Yun Lai and Zhaoqing Zhang \\ Physics Department, HKUST
}




\section{Exotic Solids Made by Elastic Metamaterials}

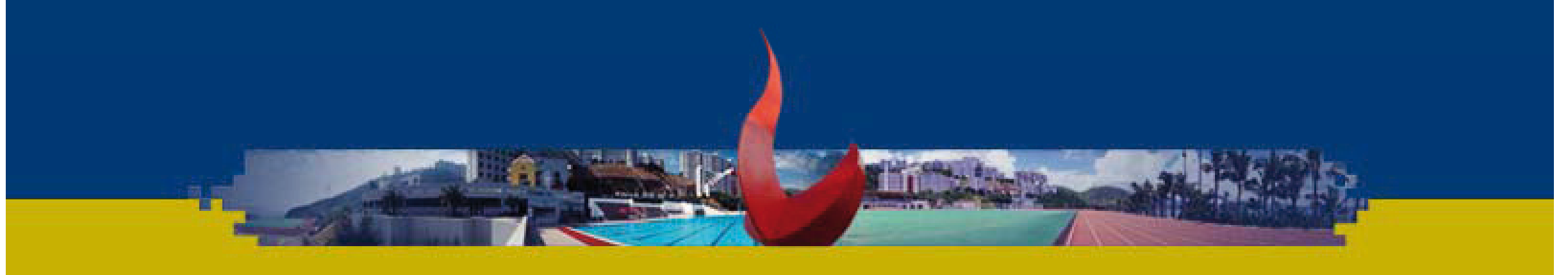

Ying Wu, Yun Lai and Zhaoqing Zhang

Physics Department, HKUST

July $13^{\text {th }}, 2010$ 


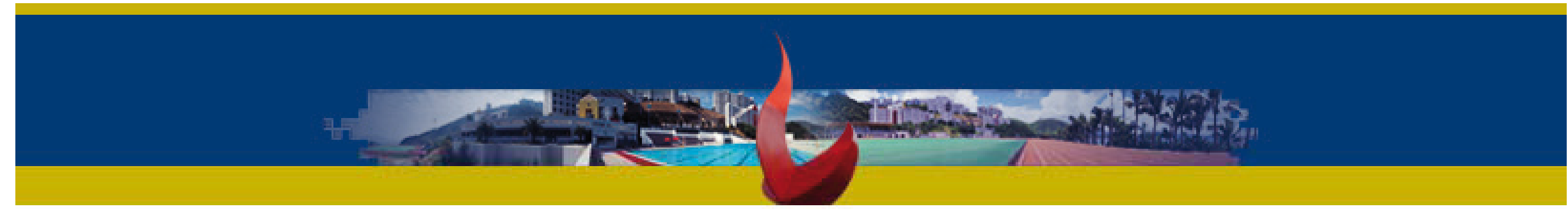

\section{What are metamaterials?}

Metamaterials are artificially structured materials that can have profoundly unique electromagnetic or optical properties.

http://people.ee.duke.edu/ drsmith/metamaterials.html

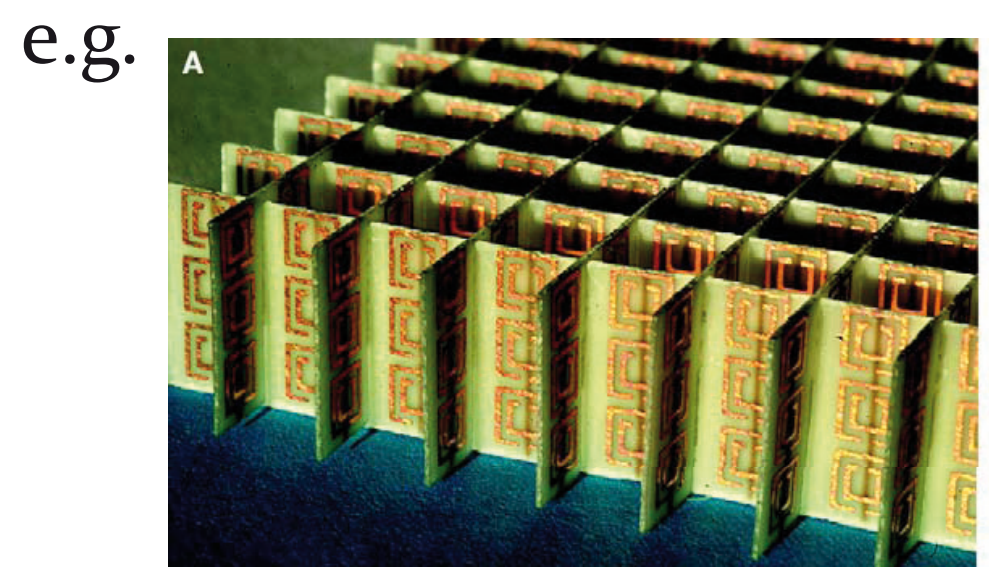

negative refraction

R. A. Shelby et al., Science 292, 77 -79 (2001)

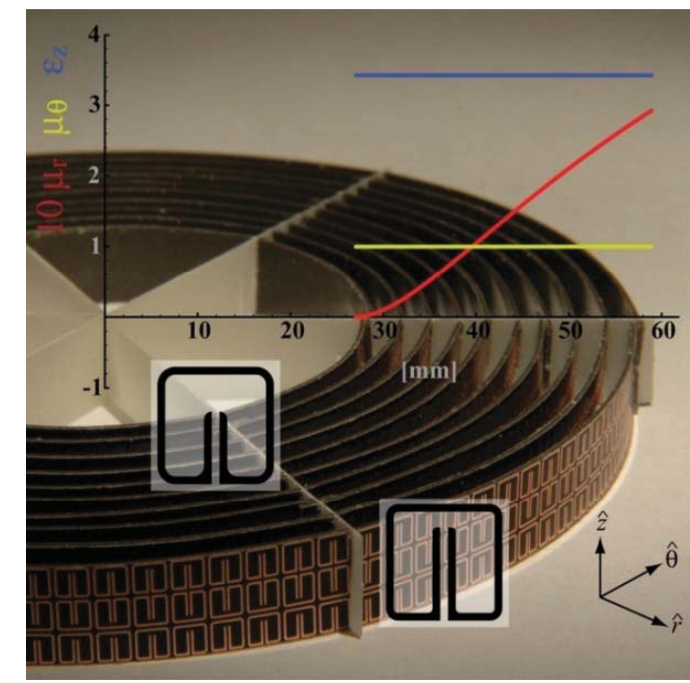

cloaking

D. Schurig et al., Science 314, 977 -980 (2006) 


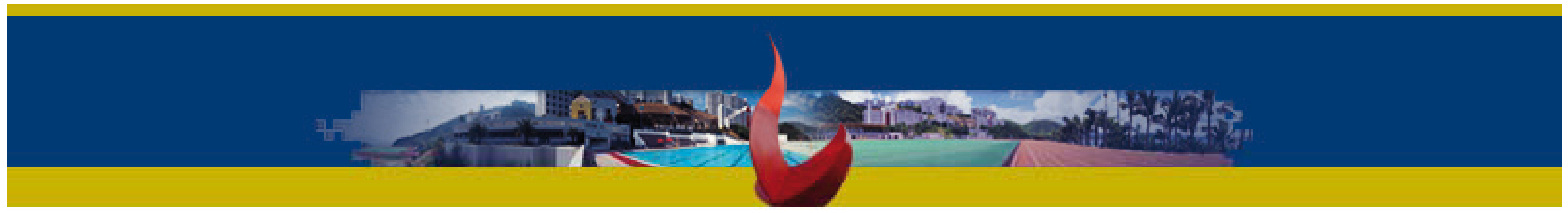

\section{Metamaterials and Effective Medium Theory}

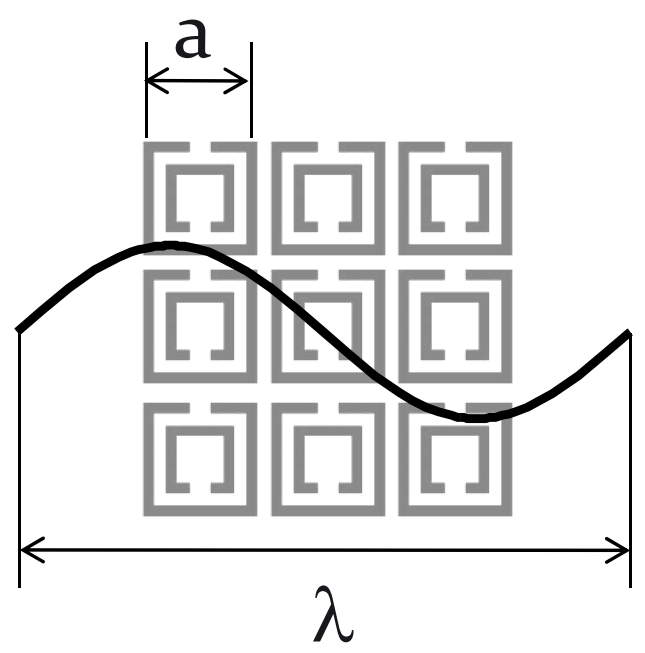

Microstructures
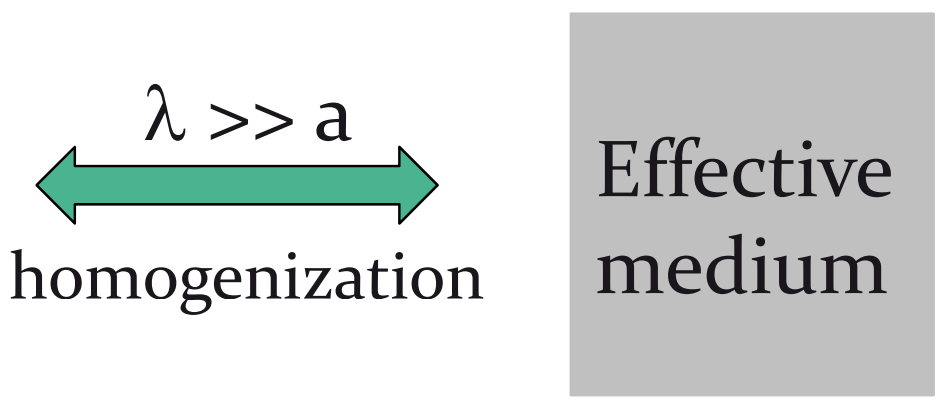

Material parameters, $(\varepsilon, \mu)$, that can describe the macroscopic properties

$$
n=\sqrt{\varepsilon} \sqrt{\mu} \longrightarrow \text { both } \varepsilon \text { and } \mu \text { are negative } \longrightarrow n<0 \text { : negative bands }
$$




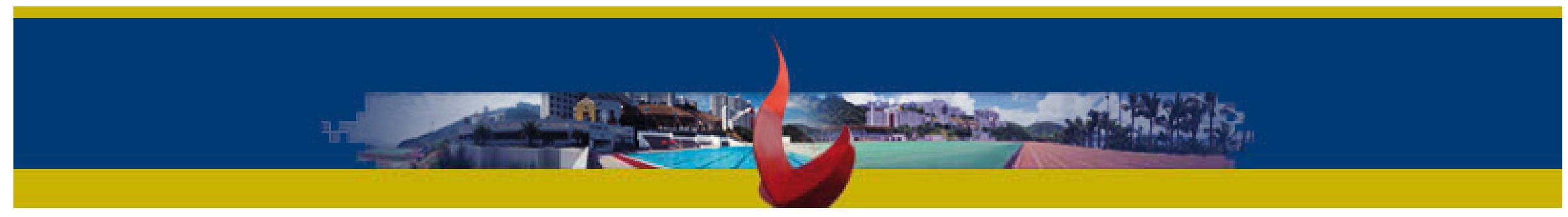

\section{What are elastic waves?}
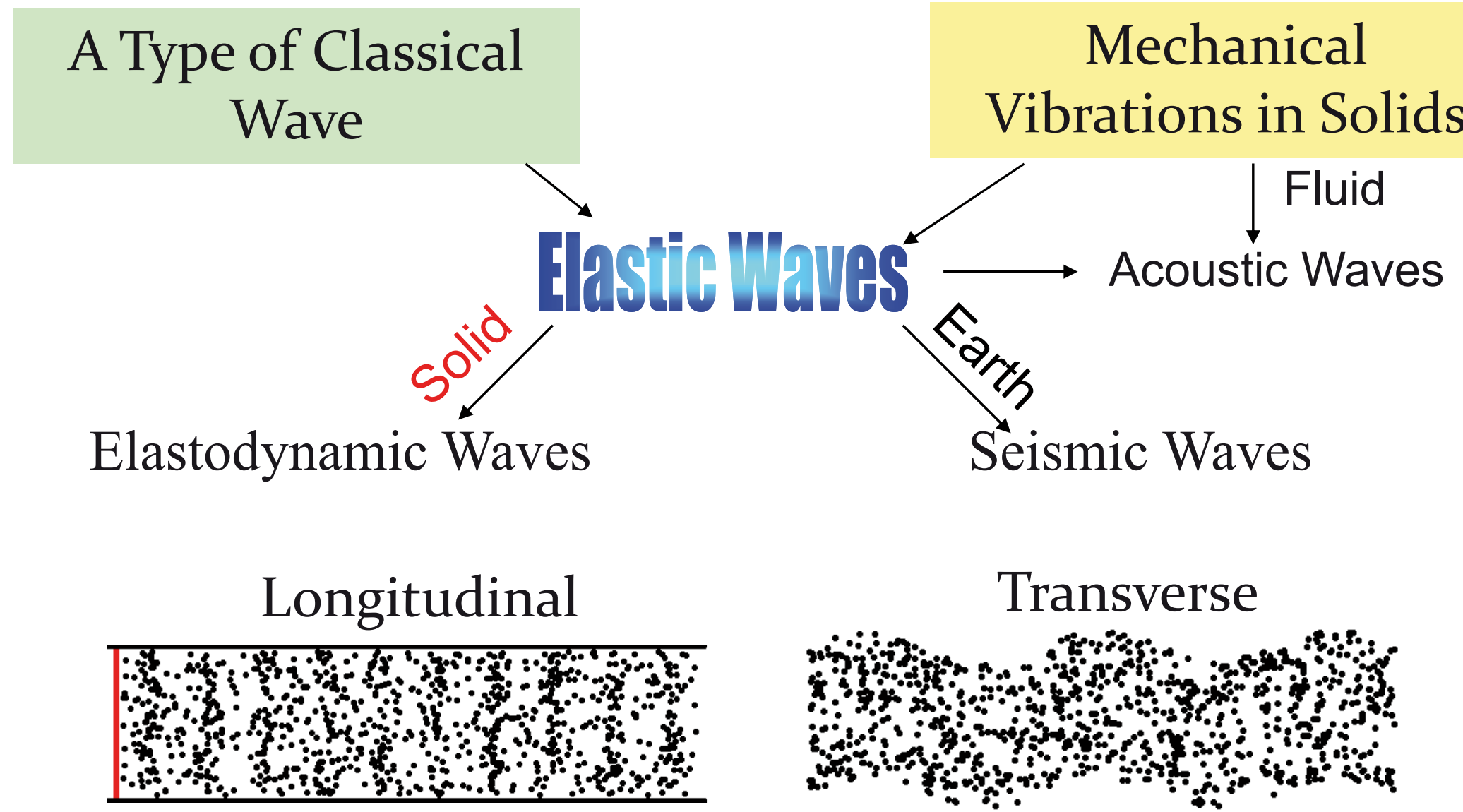


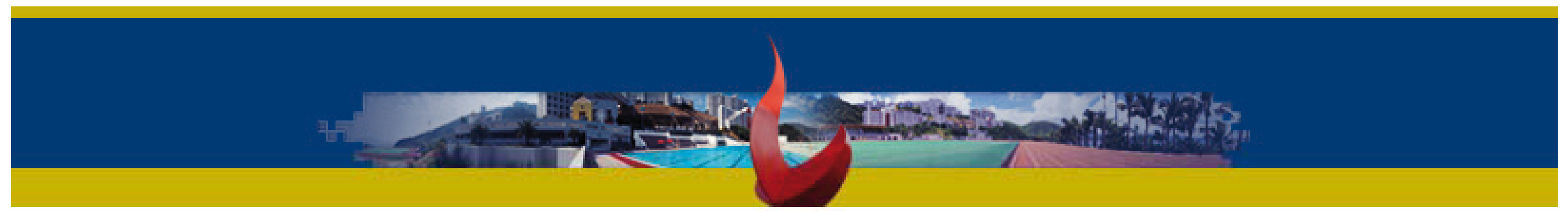

Wave equation: $\rho \frac{\partial^{2} u_{i}}{\partial t^{2}}=\nabla \cdot(\underbrace{\mu}_{\downarrow} \nabla u_{i})+\nabla \cdot\left(\mu \frac{\partial \vec{u}}{\partial x_{i}}\right)+\frac{\partial}{\partial x_{i}}\left(\sum_{\downarrow}^{\lambda \nabla} \cdot \vec{u}\right)$ mass density shear modulus lamé constant $\mu=0$ for liquids bulk modulus $\kappa=\lambda+\frac{2}{d} \mu$

Two types of waves \& three material parameters.

$$
\text { longitudinal : } n_{l}=\sqrt{(\lambda+2 \mu) / \rho} \quad \text { transverse : } n_{t}=\sqrt{\mu / \rho}
$$

\begin{tabular}{ccccc}
\hline & $\kappa_{e}+\mu_{e}>0$ & $\kappa_{e}+\mu_{e}<0$ & $\kappa_{e}+\mu_{e}>0$ & $\kappa_{e}+\mu_{e}<0$ \\
$\mu_{e}>0$ & $\mu_{e}>0$ & $\mu_{e}<0$ & $\mu_{e}<0$ \\
\hline$\rho_{e}>0$ & $n_{l e}>0, n_{t e}>0$ & $l:$ gap,$n_{t e}>0$ & $n_{l e}>0, t:$ gap & $l, t:$ gap \\
$\rho_{e}<0$ & $l, t:$ gap & $n_{l e}<0, t:$ gap & $l:$ gap,$n_{t e}<0$ & $n_{l e}<0, n_{t e}<0$ \\
\hline
\end{tabular}




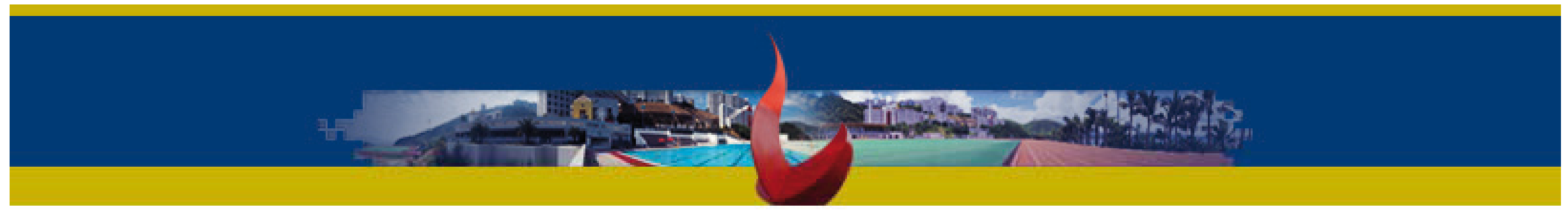

Previously proposed elastic metamaterials:

(a) $\rho<0, \kappa>0$ and $\mu>0$ (gap)

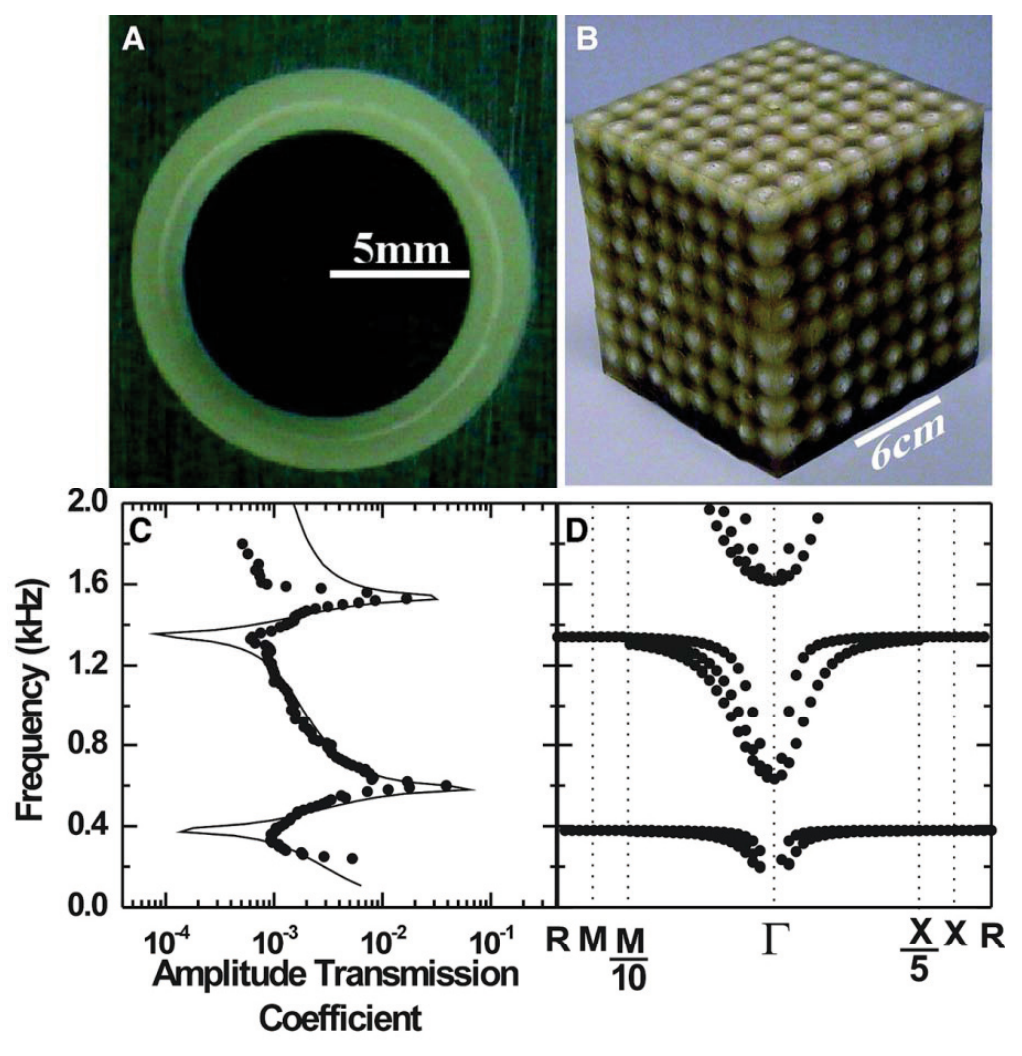

dipolar resonance

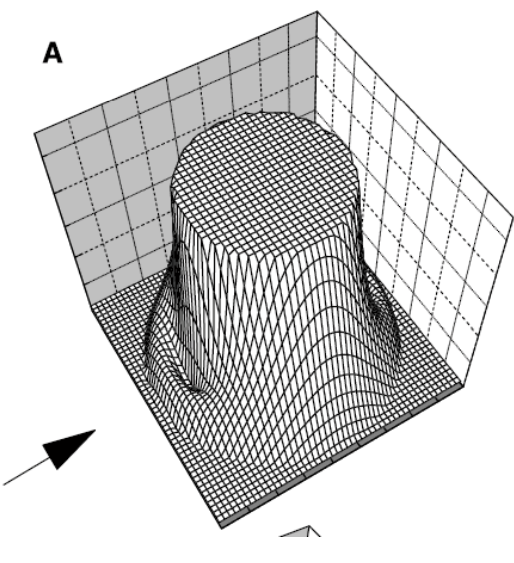

Z. Liu et al., Science 289, 1734 (2000)

Mesoscopic Physics in Complex Media 


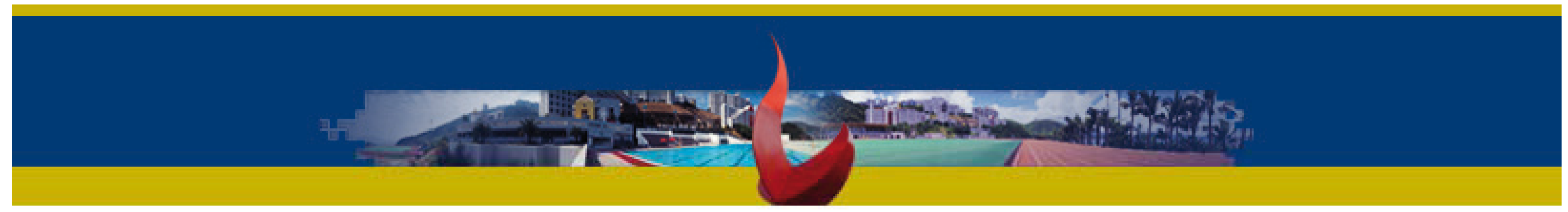

$$
\text { (b) } \rho<0, \kappa<0, \mu>0\left(n_{l}<0\right)
$$
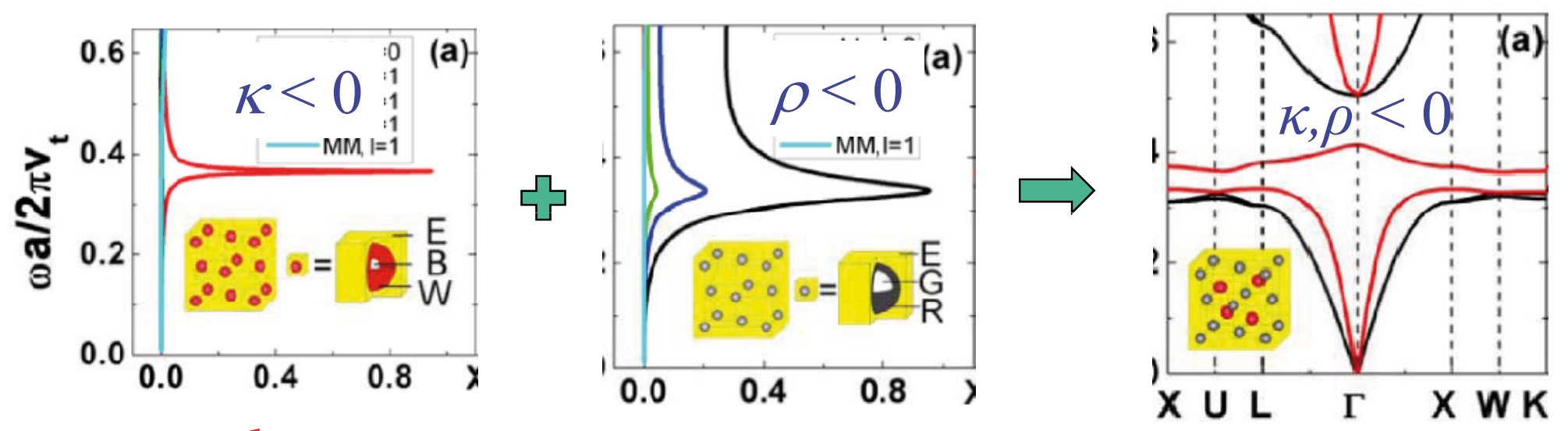
monopolar resonance

Y. Q. Ding et al., Phys. Rev. Lett. 99, 093904 (2007) 


\section{Motivation}

How about $\mu<0$ ?

\begin{tabular}{|c|c|c|c|c|}
\hline & $\begin{array}{l}\kappa_{e}+\mu_{e}>0 \\
\mu_{e}>0\end{array}$ & $\begin{array}{l}\kappa_{e}+\mu_{e}<0 \\
\mu_{e}>0\end{array}$ & $\begin{array}{l}\kappa_{e}+\mu_{e}>0 \\
\mu_{e}<0\end{array}$ & $\begin{array}{l}\kappa_{e}+\mu_{e}<0 \\
\mu_{e}<0\end{array}$ \\
\hline$\rho_{e}>0$ & $n_{l e}>0, n_{t e}>0$ & $l: g a p, n_{t e}>0$ & $n_{l e}>0, t: g a p$ & $l, t: g a p$ \\
\hline$\rho_{e}<0$ & $l, t: g a p$ & $<\underline{n}_{l e}<0, t: g a p$ & $C I: \underline{g} a$ & $\bar{n}_{l e}<0, n_{t e}<\overline{0}>$ \\
\hline
\end{tabular}

We will design "exotic solids" with $\mu<0$, also with $\rho<0, \kappa<0$ :

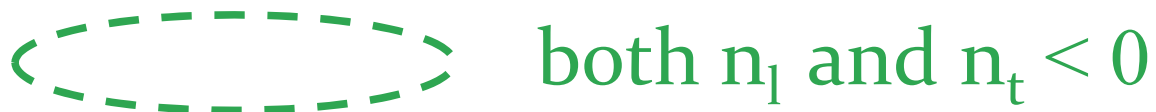

"Incompressible-solid-like": only support transverse wave

(onges 


\section{Methods}

- Effective medium analysis : guiding the design

- Numerical calculations

- Multiple-scattering calculation

- COMSOL Multiphysics 
Effective medium theory for elastic metamaterials:

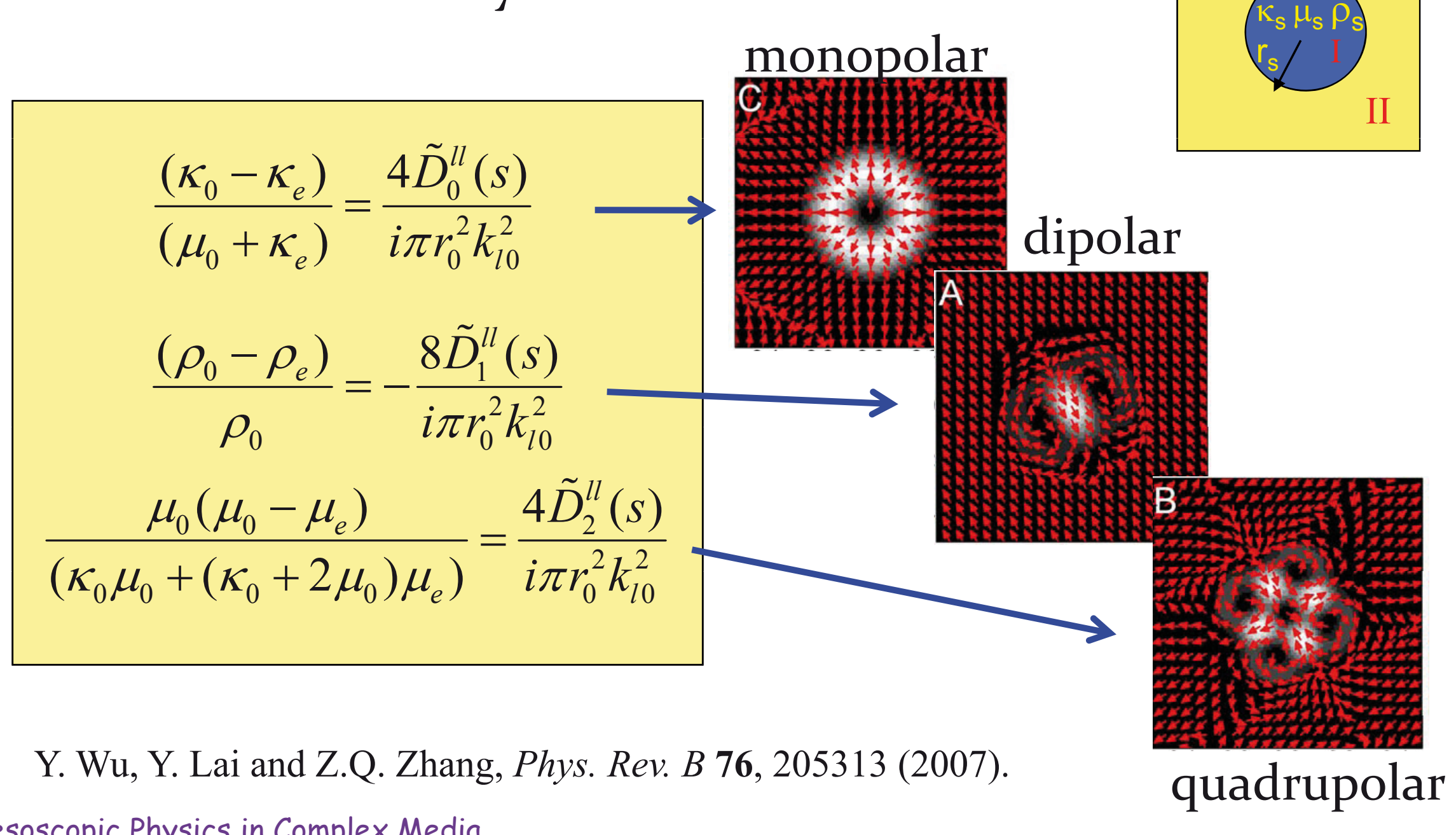




\section{Exotic soliehs}

$\mu<0, \rho<0$

Microstructure

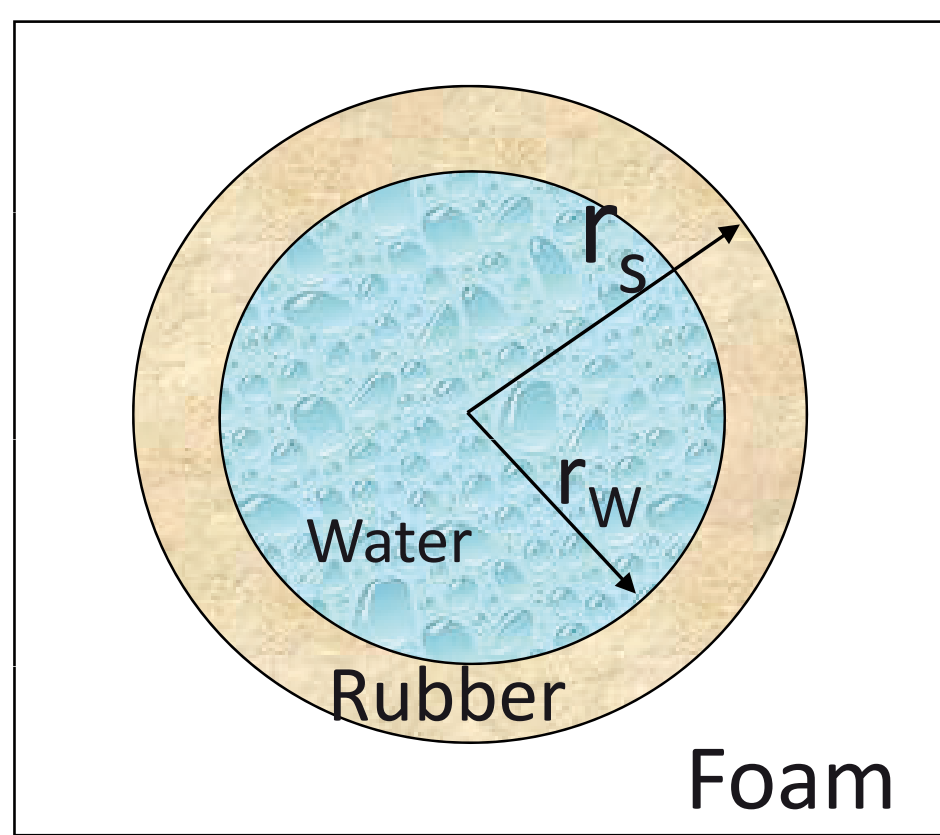

$r_{s}=0.32 a r_{w}=0.24 a$
Effective medium parameters

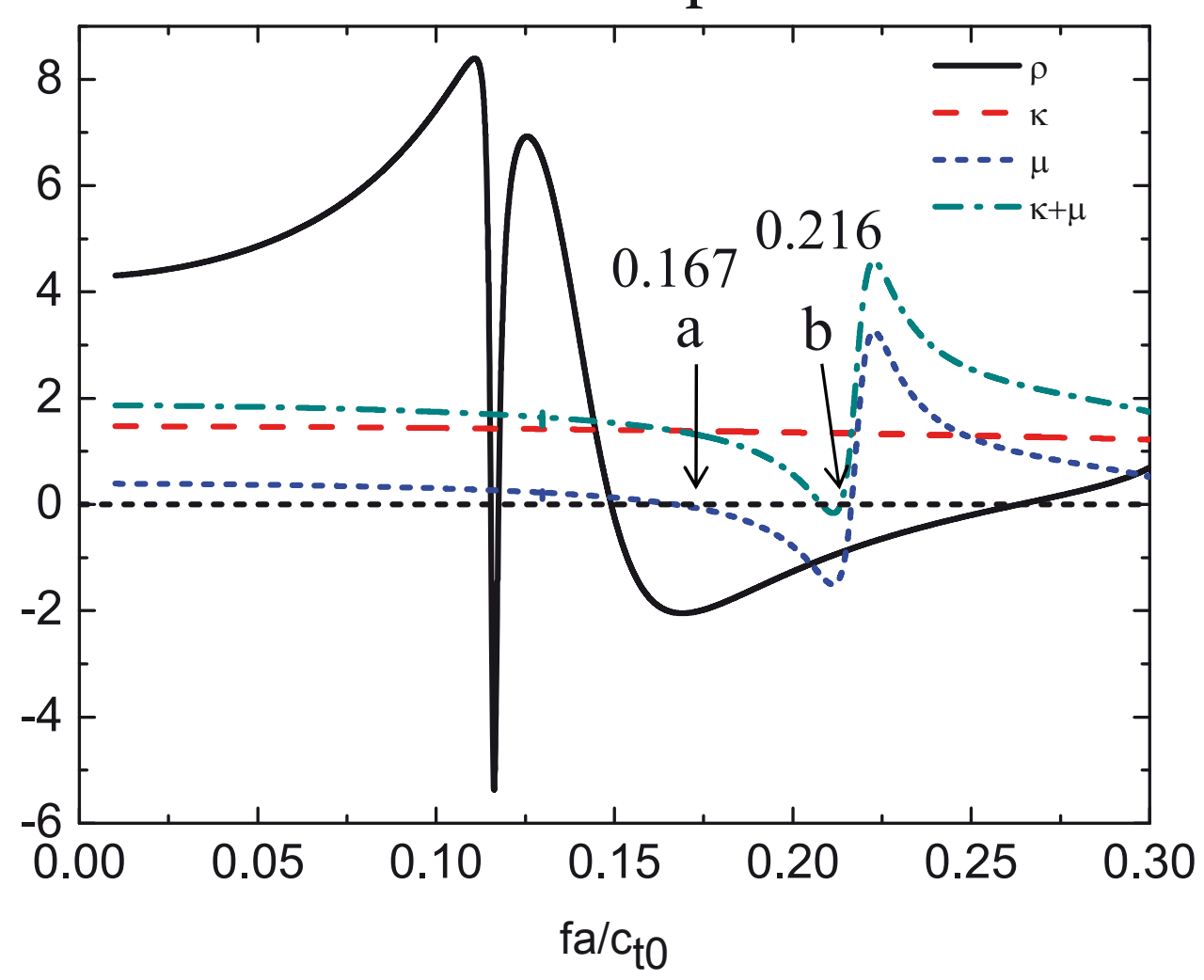




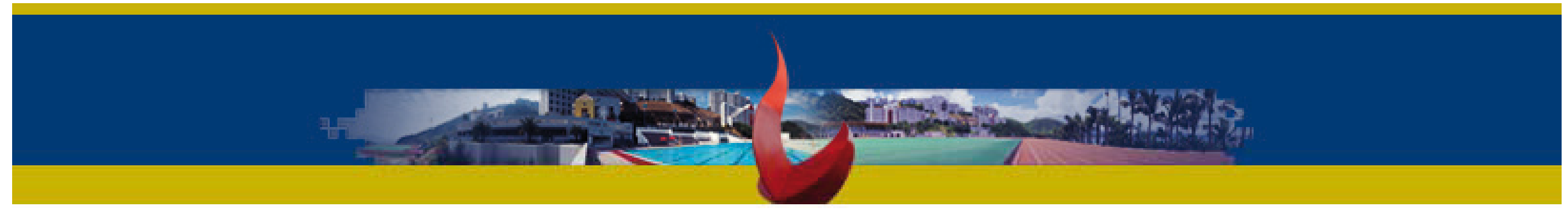

Band structure and transmission

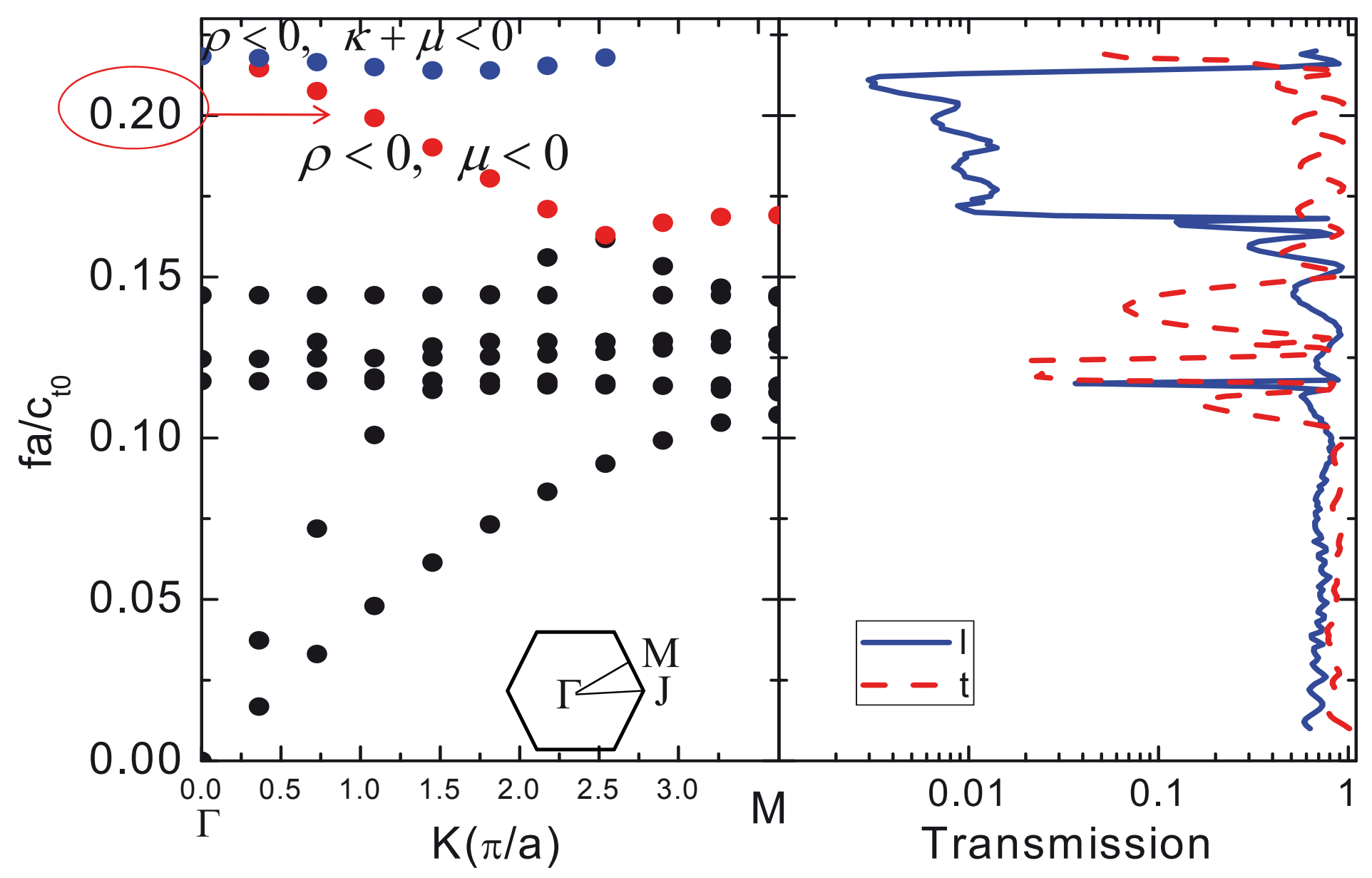




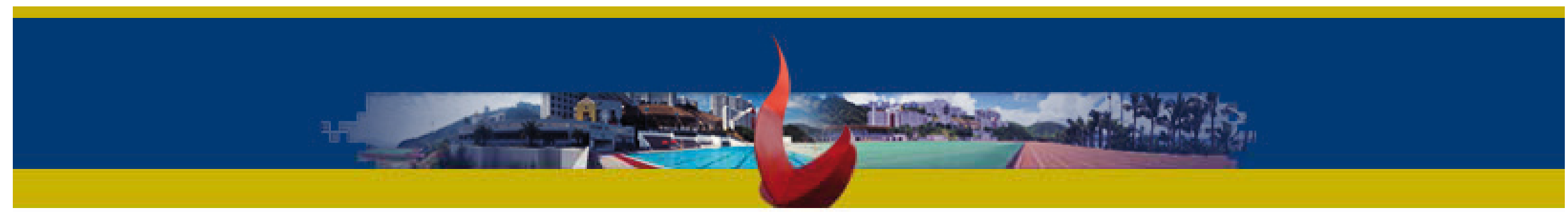

longitudinal in.
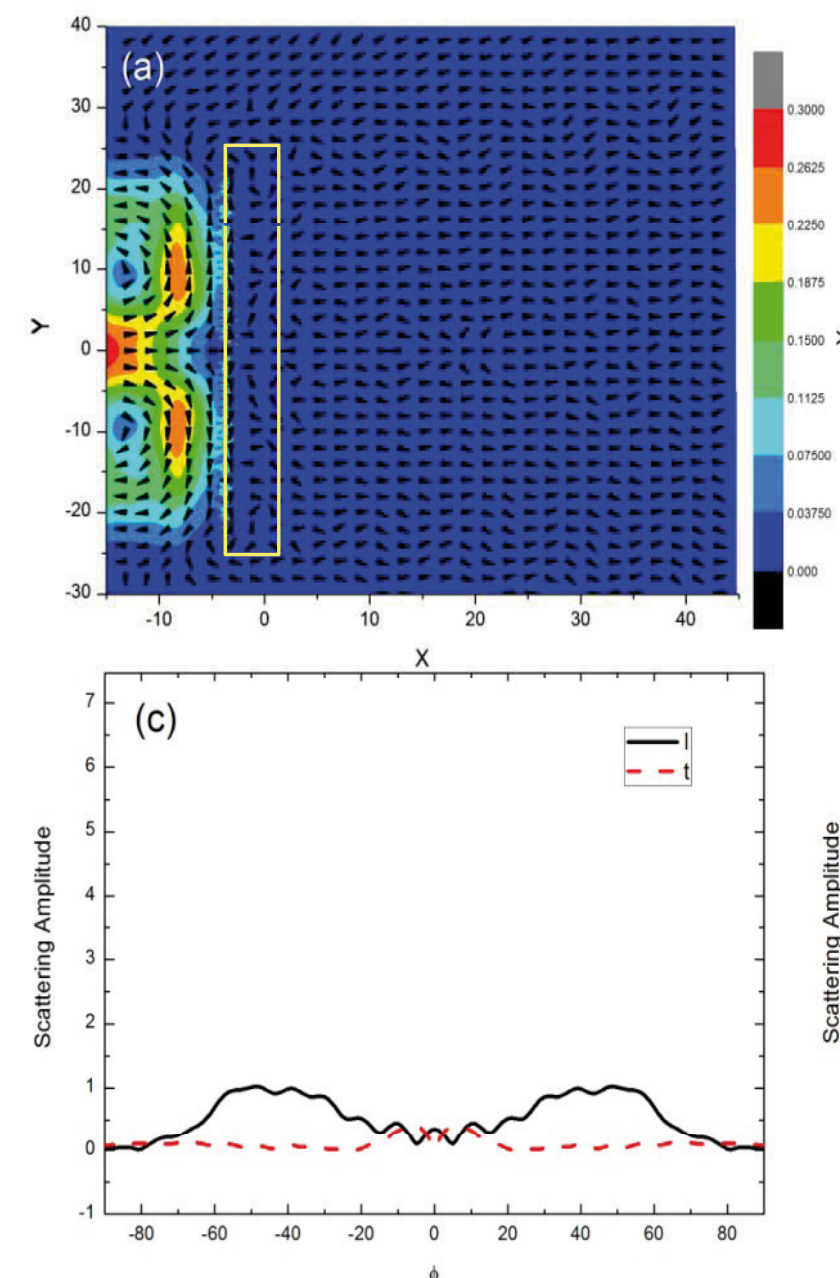

transverse in.
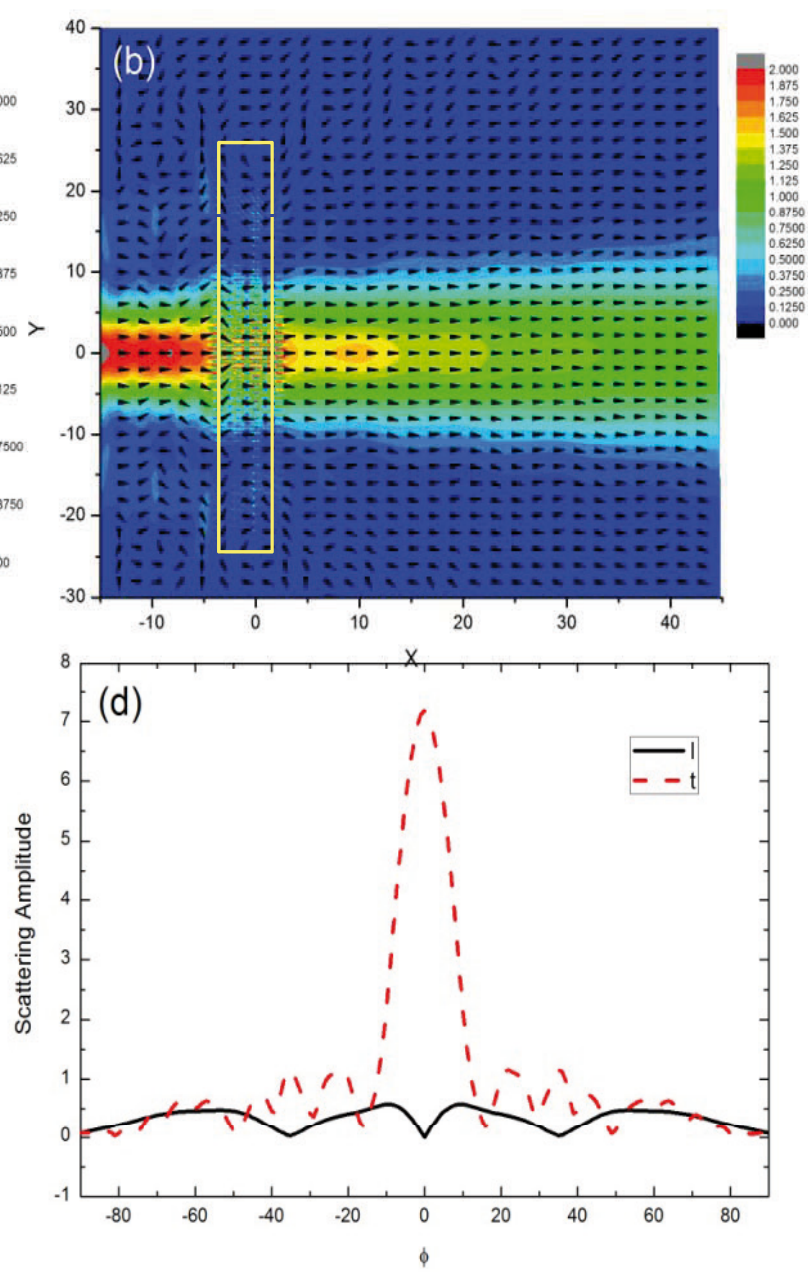

transverse wave filter "incompressi ble-solidlike" 


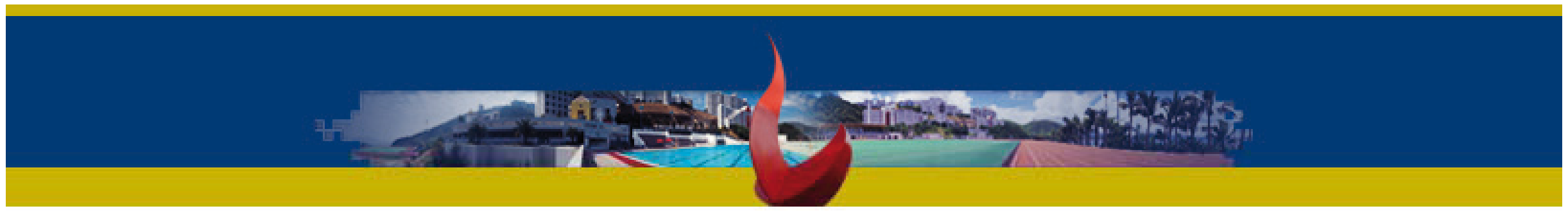

\section{negative refraction}
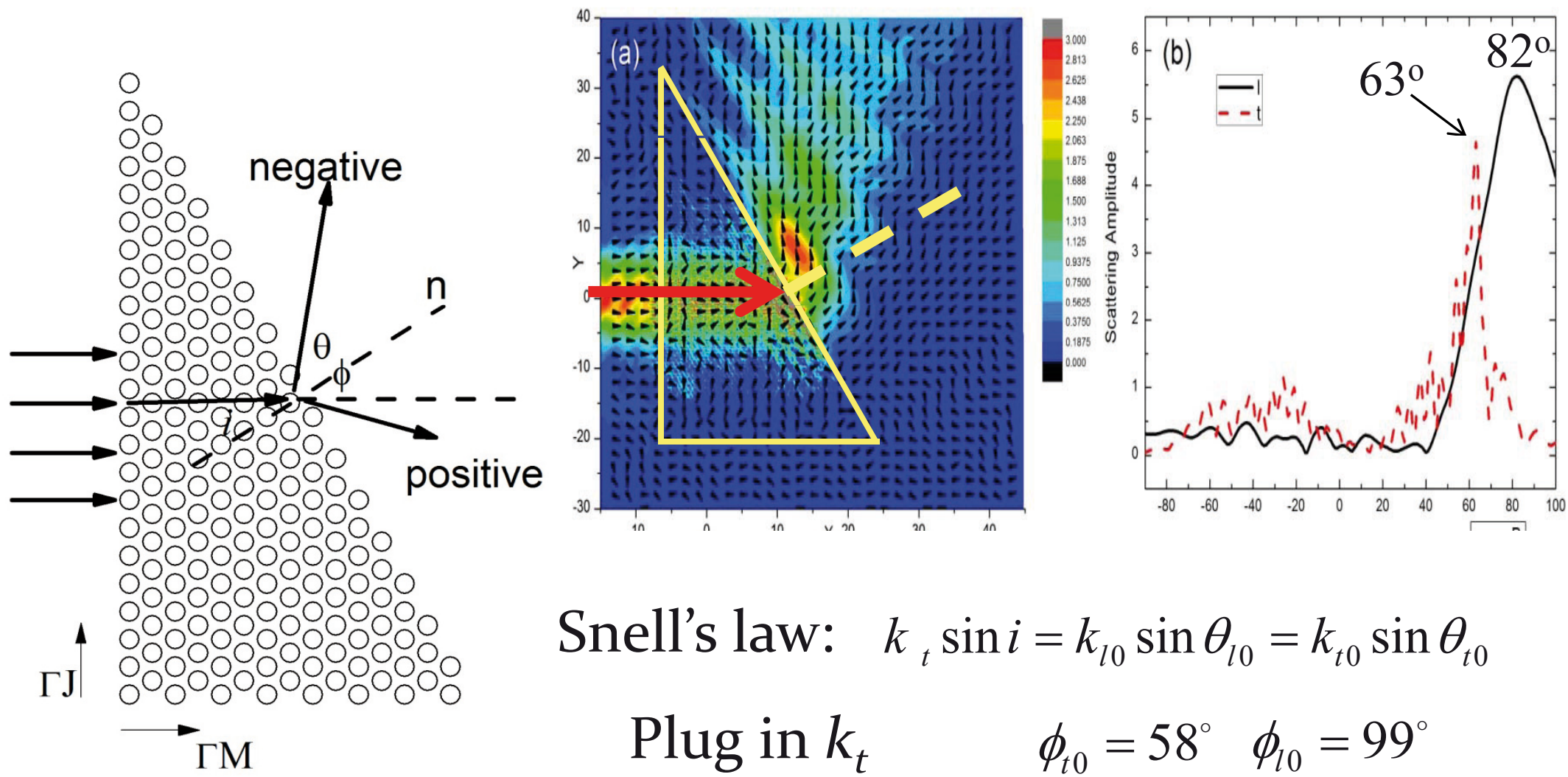

Snell's law: $k_{t} \sin i=k_{l 0} \sin \theta_{l 0}=k_{t 0} \sin \theta_{t 0}$

Plug in $k_{t} \quad \phi_{t 0}=58^{\circ} \quad \phi_{10}=99^{\circ}$ 


\section{Exotic soliets II}

From single mass LRM

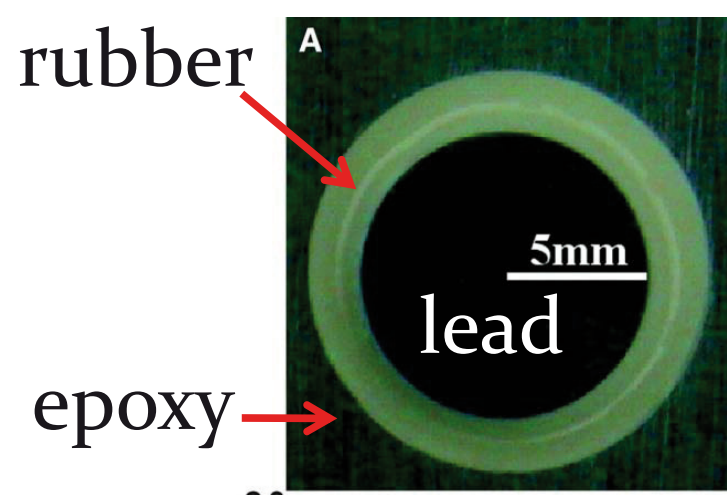

to...

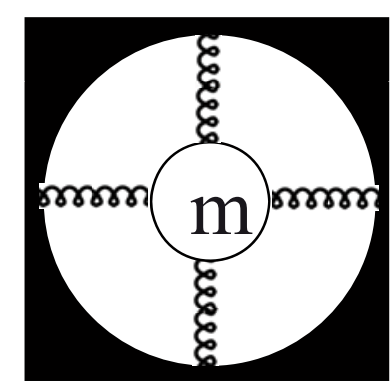

Hard core enhances the dipolar resonances which can induce negative mass density

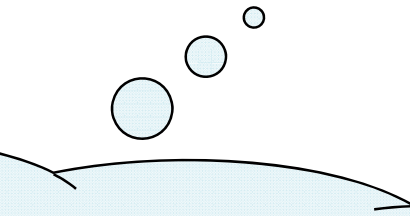

Can we use many hard objects to enhance quadrupolar resonances? $>$ Multi-mass locally resonant materials 


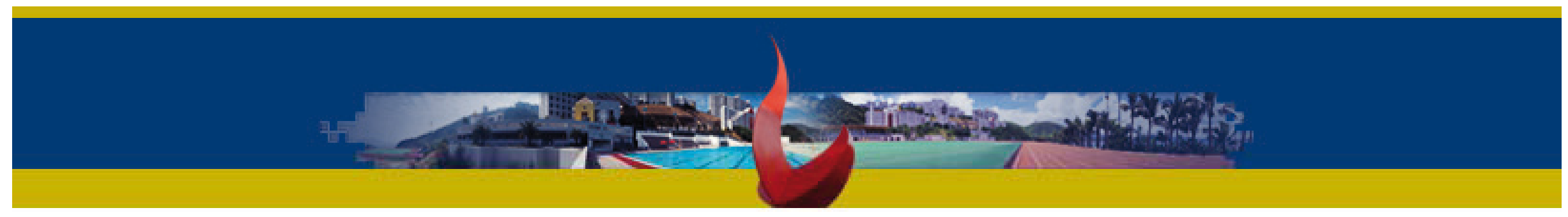

\section{System design}
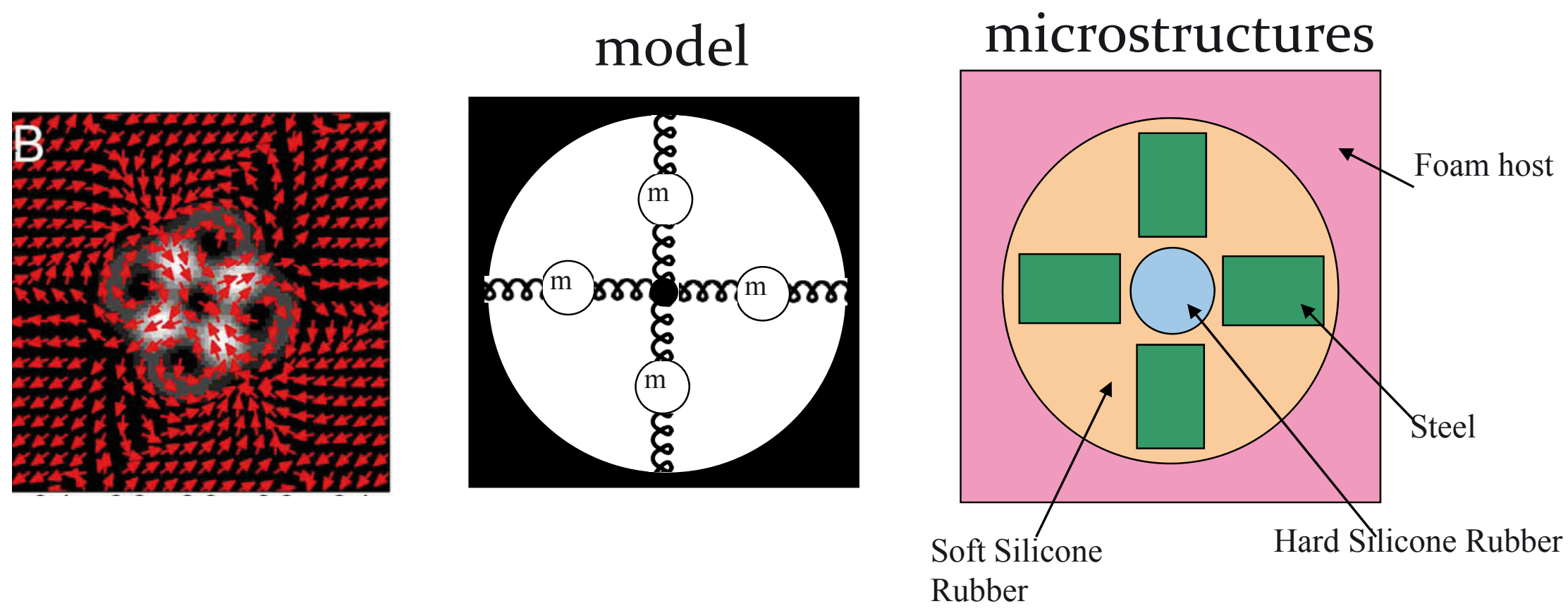

can enhance both monopolar and quadrupolar resonances 


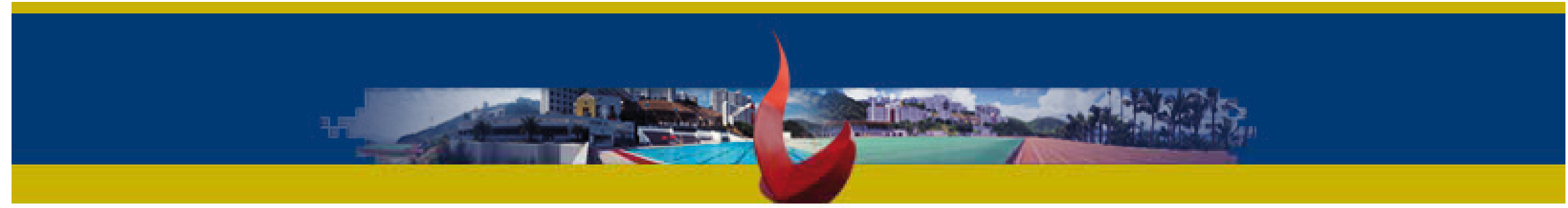

Band structure and states

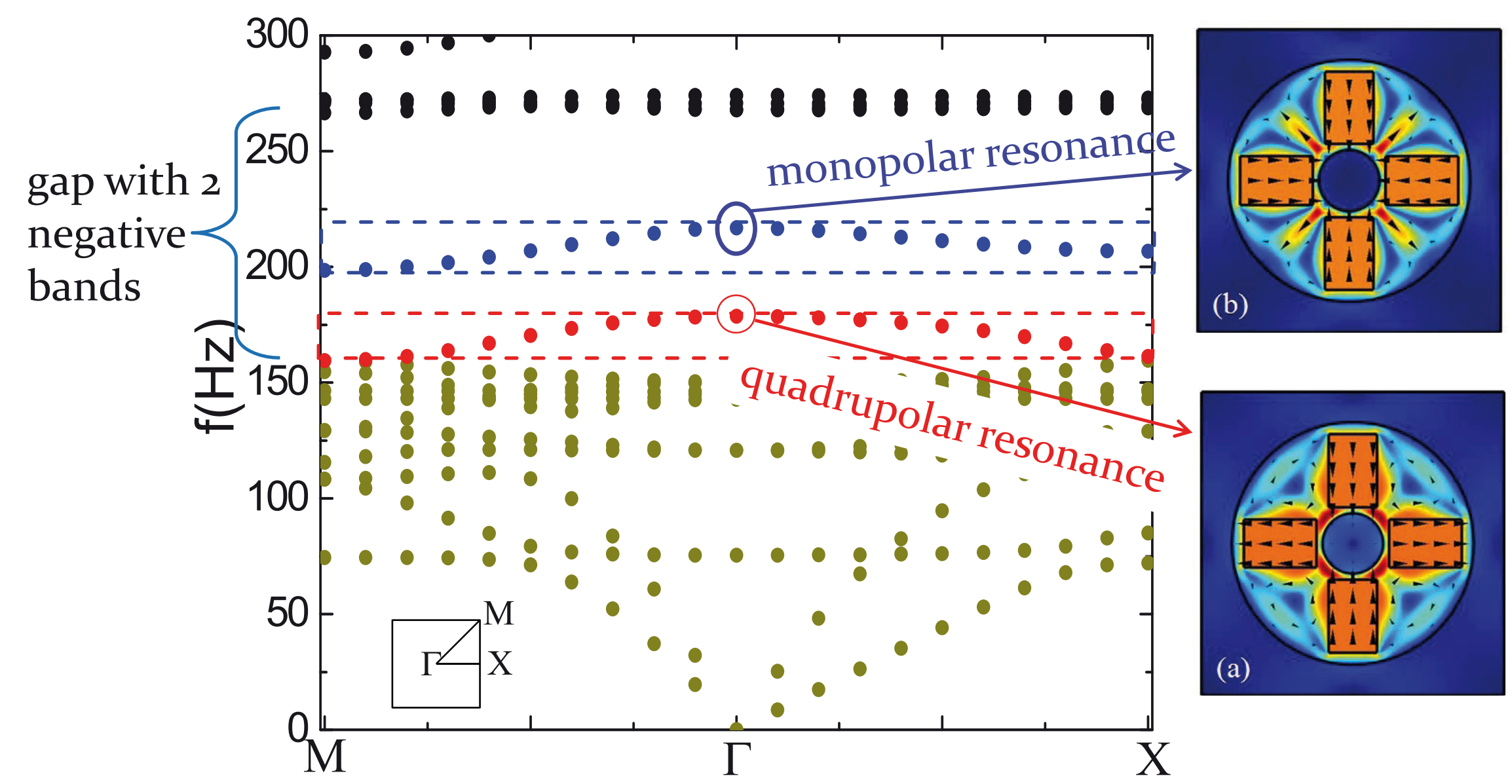



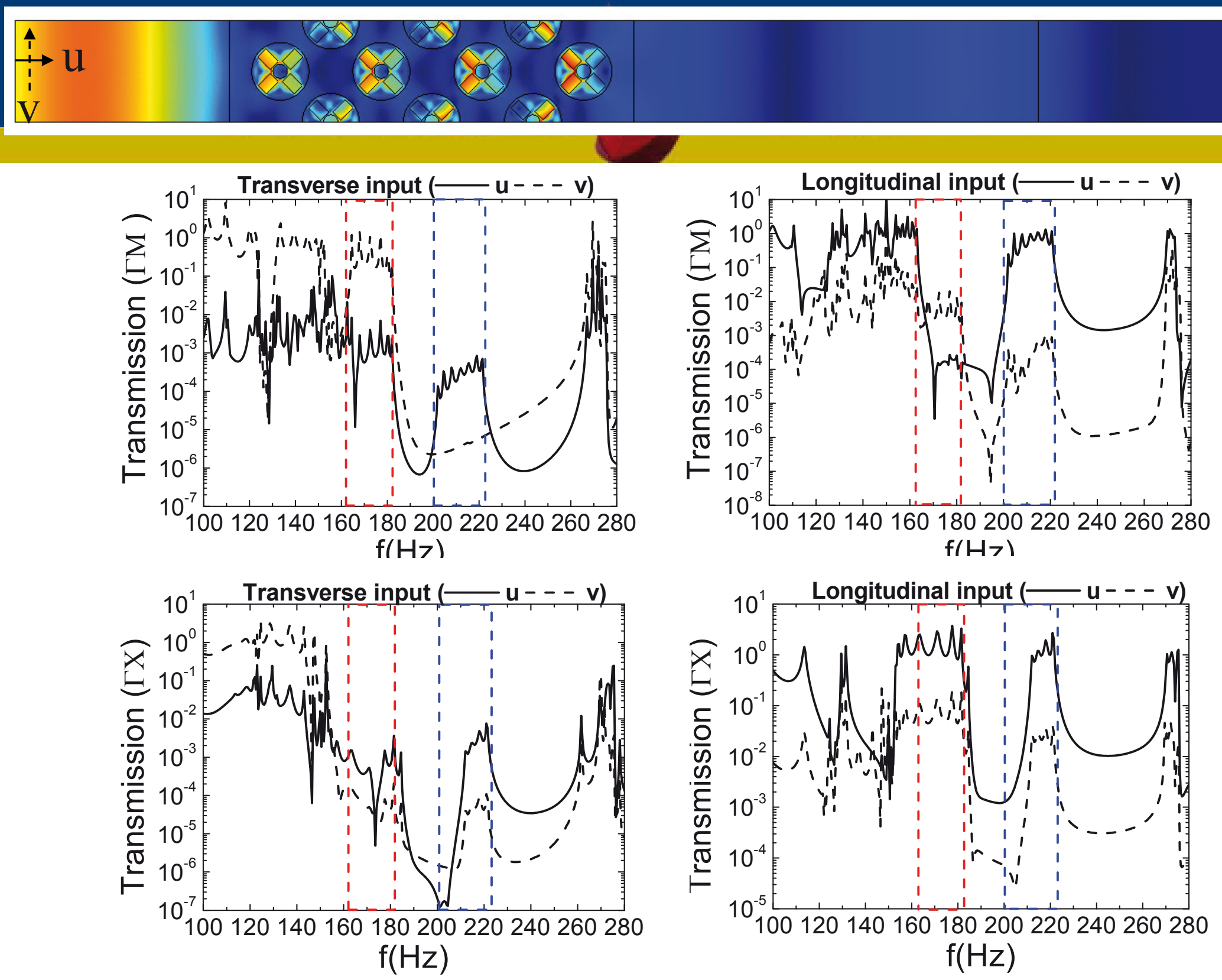

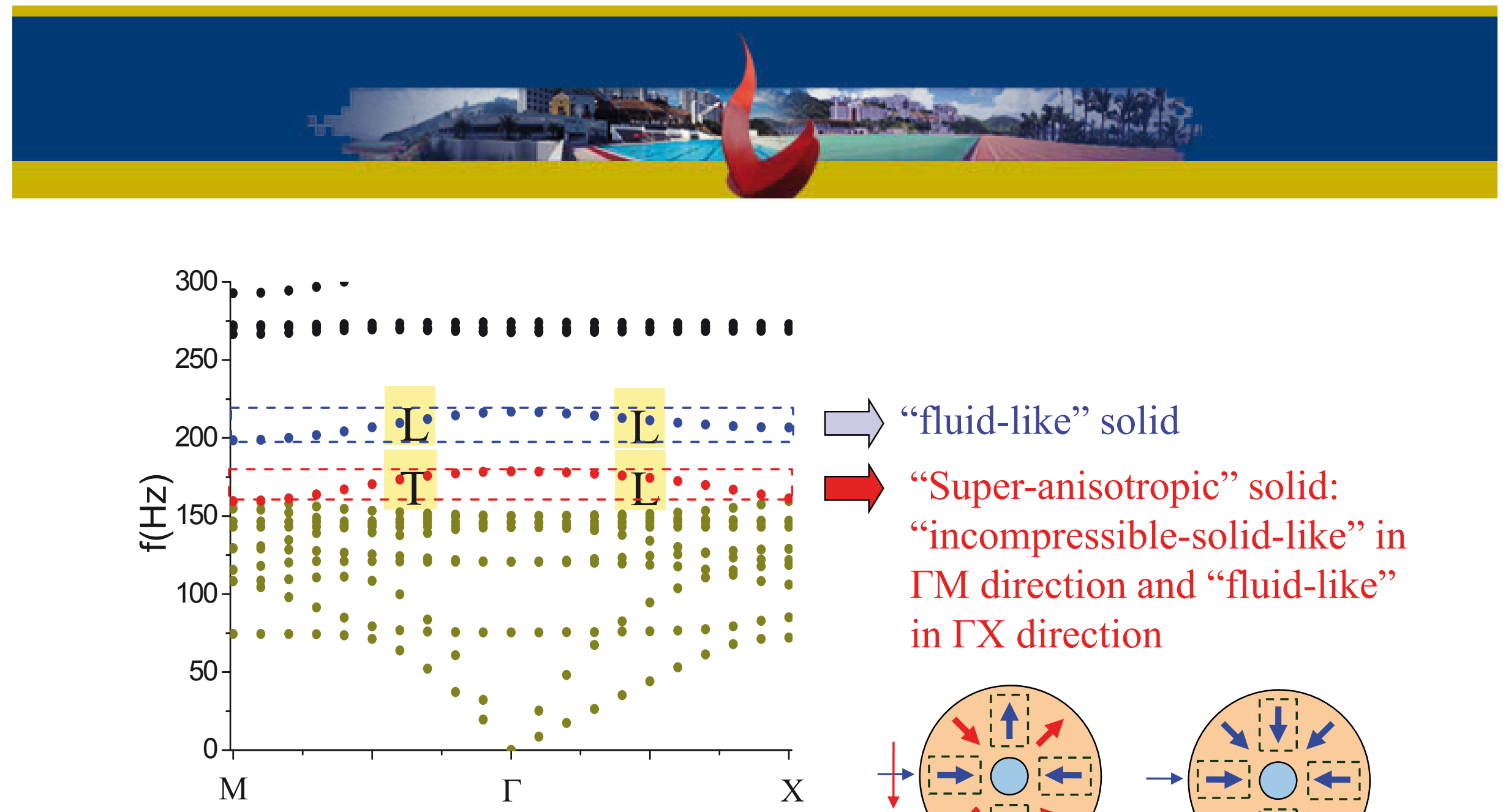

Near $\mu$ resonance, $\mu<<0, \kappa+\mu<<0$

$\Longrightarrow$ "fluid-like" solid

$\Rightarrow$ "Super-anisotropic" solid: "incompressible-solid-like" in $\Gamma \mathrm{M}$ direction and "fluid-like" in $\Gamma \mathrm{X}$ direction
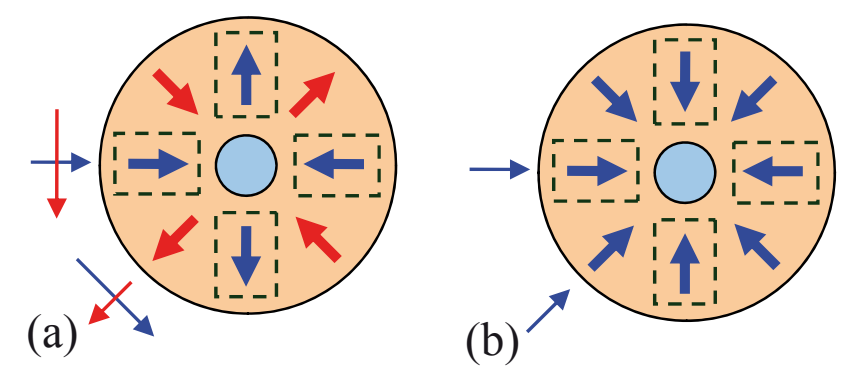

If $\rho<0$, we get $c_{l}<0$ and $c_{t}<0$, i.e. two degenerate states at $\mu=-\infty$.

Each state is longitudinal and transverse in different directions.

Symmetry breaking chooses one of them $=>$ "super-anisotropic". 


\section{Conclusions}

$>$ Design elastic metamaterials in two dimensions

$>$ guided by the effective medium theory

$>$ enhance quadrupolar resonance to produce negative $\mu$

$>$ Exotic solids (two examples):

1) Rubber-coated-water cylinders in a foam host would produce negative bands for shear wave

2) Multi-mass locally resonant structures would generate complex bands 


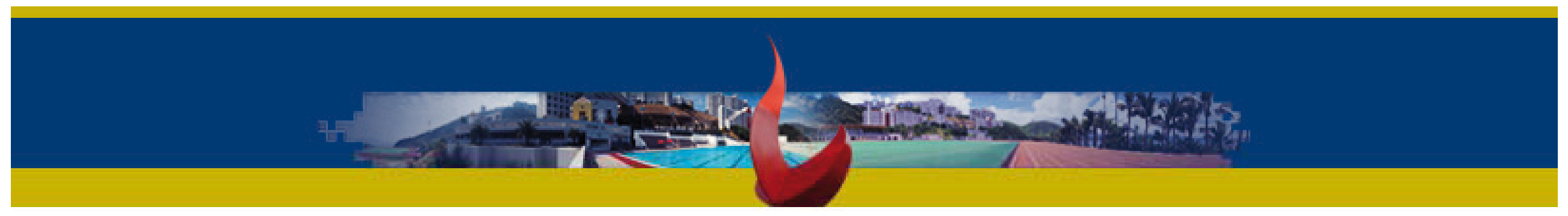

\section{The End}

